\title{
Experimental Study of Inverted L-, T-, and Related Transmission-Line Antennas ${ }^{1}$
}

\author{
Sheila Prasad and Ronold W. P. King \\ Contribution from Gordon McKay Laboratory, Harvard University, Cambridge, Mass.
}

(Received December 12, 1960; revised January 9, 1961)

\begin{abstract}
An experimental study is made of inverted L-, T-, and related transmission-line antennas and of antennas with two-, three-, and four-element top loads. The apparent measured impedance is corrected for end effects; the approximate theoretical impedances are calculated for very small heights for which measured values cannot be obtained readily. The transmission-line antennas studied are shunt-driven with one open and one closed end or with two closed ends.
\end{abstract}

\section{Introduction}

The inverted L-antenna and T-antenna, both well known in radio broadcasting, have recently found application in telemetry for missiles. Moreover, a variety of structures that are useful as missile antennas may be analyzed by an appropriate superposition of different inverted L-or $T$-antennas with images. Theories of the inverted L-antenna and T-antenna with images have been developed by Harrison [1958b; 1958d], and by King and Harrison [1959a; 1959b]; they have been extended to related circuits by Harrison [1958a; 1958c]. Special forms of these structures with low silhouette so that they may be useful as externally carried missile antennas have been studied by King, Harrison, and Denton [1958] in terms of an equivalent shunt-driven transmission line that is terminated at each end in a reactor of arbitrary value.

In order to supplement the extensive theoretical work, an experimental study has been made of the impedances of antennas of these types including especially structures with low silhouette. Antennas with multi-element top loads have also been investigated. The series of measurements was begun with a half dipole over a conducting plane. Antennas with low silhouette were approached by successively folding over larger and larger sections parallel to the ground plane, while the overall length was kept constant.

The driving-point impedances of the antennas were measured with a slotted coaxial line as shown in figure 1 for the inverted L-antenna; the ground screen was of aluminum approximately 10 by 12 feet in size. A $300 \mathrm{Mc} / \mathrm{s}$ signal with modulation at $1000 \mathrm{c} / \mathrm{s}$ was used. The detecting system included a movable voltage probe and a superheterodyne receiver.

1 This research was supported in part by the Sandia Corporation, Albuquerque, N. Mex., and the Office of Naval Research, under Contract Nonr 1866(26).

This paper first appeared in a more detailed version as a Sandia Corporation Technical Memorandum SCTM 82-60 (14) March 30, 1960.

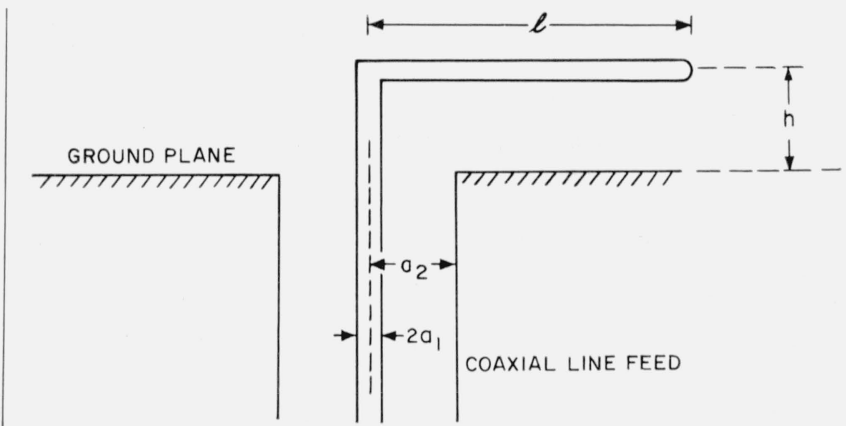

Figure 1. Inverted L-antenna.

\section{Theory of Measurement}

The theory of measurement summarized here applies to all of the experimental work described in this paper.

\subsection{Distribution Curve Method for Determining Apparent Terminal Impedance}

The part of the voltage distribution curve near a minimum was plotted and used to locate the minimum accurately. The complex terminal function $\theta_{s a}=\rho_{s a}+j \Phi_{s a}$ of the load $Z_{s a}$ is defined [King, 1955, p. 102, eq. 1] as follows:

$$
\theta_{s a}=\operatorname{coth}^{-1}\left(Z_{s a} / Z_{c}\right)
$$

where $Z_{c}$ is the characteristic impedance. The phase function $\Phi_{s a}$ is determined experimentally from the relation

$$
\Phi_{s a}=2 \pi d / \lambda+\pi / 2
$$

where $d$ is the distance between the location of the minimum with a short circuit terminating the coaxial line $\left(Z_{s a}=0\right)$ and the location of the minimum 
with the actual load $Z_{s a}$ terminating the line. The attenuation function $\rho_{s a}$ is obtained from

$$
\sinh ^{2} \rho_{s a}=\frac{\sin ^{2}\left(\beta W_{2} / 2\right)-\frac{p_{2}^{2}}{p_{1}^{2}} \sin ^{2}\left(\beta W_{1} / 2\right)}{\frac{p_{2}^{2}}{p_{1}^{2}}-1}
$$

where $W_{1}$ and $W_{2}$ are the widths of the distribution curve minima at the power levels $p_{1}^{2}$ and $p_{2}^{2}$ [King, 1952]. With a square-law detecting system, the power levels $p_{1}^{2}$ and $p_{2}^{2}$ could be read directly on a Ballantine voltmeter. Since $\rho_{s a}$ is small in all cases, the approximation $\sinh ^{2} \rho_{s a} \doteq \rho_{s a}$ is valid. From the experimentally determined values of $\rho_{s a}$ and $\Phi_{s a}$, the apparent admittance $Y_{s a}=G_{s a}+j B_{s a}$ may be calculated with the following formulas [King, 1955, p. 102, eq. 12]:

$$
\begin{aligned}
g_{s a} & =\frac{\sinh 2 \rho_{s a}}{\cosh 2 \rho_{s a}+\cos 2 \Phi_{s a}} \\
b_{s a} & =\frac{\sin 2 \Phi_{s a}}{\cosh 2 \rho_{s a}+\cos 2 \Phi_{s a}}
\end{aligned}
$$

where the normalized admittance is

$$
y_{s a}=Y_{s a} Z_{c}=g_{s a}+j b_{s a} .
$$

The apparent impedance of the load is $Z_{s a}=1 / Y_{s a}$. $Z_{c}$ is the characteristic impedance of the coaxial line; its numerical value is $Z_{c}=60.06$ ohms.

\subsection{End Correction for the Coaxial Line Driving the Antenna Over a Ground Plane}

When an antenna is driven over a ground plane by a coaxial line, it is necessary to apply an end correction [King, 1955, p. 430] in order to obtain the theoretical impedance $Z_{0}$ from the measured apparent impedance $Z_{s a}$ terminating the line. This correction consists of a capacitance $C_{T c}$ in parallel with $Z_{0}=1 / Y_{0}$ so that

$$
Y_{s a}=Y_{0}+j \omega C_{T c} .
$$

A curve of $C_{T c} / c_{0} a_{2}$ as a function of $a_{2} / a_{1}$ is given by King [1955, p. 435, fig. 22.4]; $a_{2}$ and $a_{1}$ are the radii of the outer and inner conductors of the coaxial line. For $a_{2} / a_{1}=2.762, C_{T c} / c_{0} a_{2}=-0.25$ or $C_{T c}=-0.242 \times 10^{-12}$ farad. It follows from (6) that

$$
\begin{aligned}
Y_{0}=y_{0} R_{c}=\left(g_{0}+j b_{0}\right) R_{c}=Y_{s a} & -j B_{T c} \\
& =G_{s a}+j\left(B_{s a}-\omega C_{T c}\right) .
\end{aligned}
$$

The normalized theoretical impedance is

$$
z_{0}=r_{0}+j x_{0}=Z_{0} / R_{c}=1 / y_{0} .
$$

When $\beta(h+l)$ is near $\pi / 2, \omega C_{T c}$ is negligible compared with $B_{s a}$; when $\beta(h+l)$ is near $\pi$, the contribution by $\omega C_{T c}$ is significant. The theoretical terminal functions $\rho_{0}$ and $\Phi_{0}$ in $\theta_{0}=\rho_{0}+j \Phi_{0}$ are also computed from eq (1) with the subscript 0 in place of $s a$. Since $G_{0}=G_{s a}$ it follows that $\rho_{0}=\rho_{s a}$. Since the end effect is purely susceptive and $b_{0}$ is small, the terminal function is given by

$$
\Phi_{0}=\pi+\tan ^{-1} b_{0} \doteq \pi+b_{0} .
$$

\subsection{End Correction and Theory for Antennas of Small Height}

Owing to the finite size of the conductors, it was not practical in the experiment to use antennas with heights $h$ (fig. 1) less than $2.5 \mathrm{~cm}(\beta h=2 \pi h / \lambda \geqq 0.161)$. Therefore, the experimentally determined curves of $\rho_{0}$ and $\Phi_{0}$ were extended with theoretical values. These are readily determined since for small heights, the inverted L-antenna with its image is essentially a balanced section of a two-wire transmission line for which the radiation resistance referred to maximum current is given by

$$
R^{e}=30 \beta^{2}(2 h)^{2}=120 \beta^{2} h^{2} .
$$

When the maximum current is at the driving point, $\beta(h+l)=\pi / 2$, the input resistance is $R_{i n}=R^{e}$. In the case of a T-antenna with image, the resistance is one-half of $R_{i n}$ for the inverted L-antenna with its image since the two equal resistances are in parallel. In general, for the antenna with $n$-element top load with image, the resistance is $1 / n$ of $R_{i n}$ for the inverted L-antenna with its image since $n$ equal resistances are in parallel. When the overall electrical length of the inverted L-antenna is $\pi$, the radiation resistance $R^{e}$ referred to maximum current must be transformed through an electrical length of line $\beta w=\pi / 2$ to obtain the resistance at the driving point. The relevant formula is [King, 1955, p. 134 , eq $9 \mathrm{a}, \mathrm{b}] R_{\text {in }}=R_{c}^{\prime 2} / R^{e}$ where $R_{c}^{\prime}$ is the characteristic resistance of the two-wire line formed by the inverted L-antenna and its image. If $h$ denotes the height of the antenna over the ground plane (one-half the spacing of the two-wire line) and $a_{1}$ is the radius of the conductor, $R_{c}^{\prime}$ is given by

$$
R_{c}^{\prime}=120 \cosh ^{-1}\left(h / a_{1}\right) .
$$

The theoretical value of the terminal function $\rho_{0}$ may be calculated from the general formula [King, 1955, p. 102, eq 9]. When $r_{0}^{2} \gg 1, r_{0}^{2} \gg x_{0}^{2}$ this reduces to

$$
\rho_{0} \doteq 1 / 2 \tanh ^{-1} 2 / r_{0} \doteq 1 / r_{0} .
$$

For antennas with overall electrical length (height + one element of top load) equal to approximately $\pi / 2, r_{0}=R^{e} / R_{c}^{\prime}$ and $\rho_{0}=R_{c}^{\prime} / R^{e}$. When the overall electrical length is $\pi$, the maximum current is a quarter wavelength from the driving point and

$$
r_{0}=\left(R_{c}^{\prime 2} / K^{e}\right)\left(1 / R_{c}^{\prime}\right)=R_{c}^{\prime} / R^{e} .
$$

It follows that for small values of $\beta h$,

$$
\rho_{0}=R^{e} / R_{c}^{\prime} \text {. }
$$


The terminal function $\Phi_{0}$ for small $\beta h$ and when $\beta(h+l)=\pi / 2$ is obtained from that of a transmission line with input reactance $X_{i n}$ in series with the inductive reactance $X_{L}=\omega L$ of the short length of conductor of length $h$ and radius $a_{1}$ perpendicular to the ground plane. The inductance of this straight conductor is [King, 1945]

$$
L=\left(\mu_{0} h / \pi\right)\left[\ln \left(4 h / a_{1}\right)+\left(a_{1} / 2 h\right)-1\right] .
$$

The input reactance $X_{i n}$ includes a contribution from the end effect at the open end. This is approximately equivalent to a lumped capacitance $C_{T e}$ that is given by the following formula [King, 1955, p. 367, eq 13]

$$
C_{T e}=\frac{\pi \epsilon_{0}\left(2 h-a_{1}\right)}{2\left[\ln \left(2 h / a_{1}\right)^{2}\right]} .
$$

The general formula for transforming a reactance $X_{T e}=-1 / \omega C_{T e}$ from the end of a line of length $l$ to its driving point is obtained from King [1955, p. 134, eq $9 \mathrm{a}, \mathrm{b}]$ in the special case when the termination is purely reactive. The sum of the normalized reactances $x=x_{i n}+x_{L}$ (normalized with respect to $R_{c}^{\prime}$, the characteristic impedance of the two-wire line) gives the total normalized reactance terminating the coaxial line. $\Phi_{0}$ may be obtained for a purely reactive termination from (1) with appropriate change in subscript. With $x^{2} \ll 1$,

$$
\Phi_{0} \doteq \pi / 2+x \text {. }
$$

The formula gives $\Phi_{0}$ for small $\beta h$ where the overall electrical length of the antenna is $\beta(h+l) \doteq \pi / 2$.

When the antenna length is $\beta(h+l) \doteq \pi$, the current is small at the driving point and the reactance terminating the coaxial line may be assumed to be the input reactance of a section of open-end line of length $(h+l)$. The equivalent lumped capacitance of the open end, $C_{T e}$, may be transformed to the driving point, so that $x \doteq x_{T e}=X_{T e} / R_{c}^{\prime}$. In this case, since $x^{2} \gg 1$,

$$
\Phi_{0} \doteq \pi-1 / x=\pi+\omega C_{T e} .
$$

In the preceding discussion, approximate corrections have been made for reactive end effects at the load end of the coaxial line and at the open ends of the open-wire lines forming the antennas. It is to be noted that an accurate analysis of end effects when the generator is in the terminal zone is not available. In the case at hand the diameter of the coaxial line is of the same order of magnitude as the smallest heights $h$ of the antennas so that the electromagnetic field in the vicinity of the driven end of the antennas and the load end of the coaxial line is complicated. An accurate representation in terms of an equivalent lumped load for the coaxial line and equivalent point generator for the antenna is not possible and a close quantitative agreement between theoretical results based on such a representation and measured values cannot be expected in general.

\section{Experimental Results for Inverted $L_{-}, T_{-}$, and Multi-Element Top-Loaded Antennas}

The measured values of the apparent terminal functions $\theta_{s a}=\rho_{s a}+j \Phi_{s a}$ are shown in figure 2 for the inverted L-antenna with $\beta(h+l)=3.2$. The corresponding corrected functions $\theta_{0}=\rho_{0}+j \Phi_{0}$ with their theoretically derived extensions to small values of $\beta h$ are also shown. The apparent terminal impedances $Z_{s a}$ and the corrected impedances $Z_{0}=R_{0}$ $+j X_{0}$ are shown in figure 3 for the inverted L-antenna with $\beta(h+l)=3.2$. Note that $Z_{s a}$ is the impedance actually observed as terminating the coaxial line; it includes end effects. $Z_{0}$ is the ideal impedance of the antenna in the absence of end effects. Figure 4 shows the impedance of the inverted L-antenna, T-antenna, and antennas with two-, three-, and four-element top loads with $\beta(h+l)$ $=1.6$.

\section{Antennas of Transmission-Line Types}

It has been shown by King, Harrison, and Denton [1958] that antennas of transmission-line types may be treated by transmission line theory corrected for radiation if the height $h$ of the antenna is sufficiently small. A general formula for the input resistance at the driving point is given by King, Harrison, and Denton [1958]. This formula does not take account of the effect on the driving-point resistance of the conductor of length $2 h$ in series with the generator. Nor is this effect included if the inductive reactance $X_{L}=\omega L$ of the section of length $2 h$ is added to the input reactances $X_{1 \text { in }}$ and $X_{2 \text { in }}$ of the parallel com-

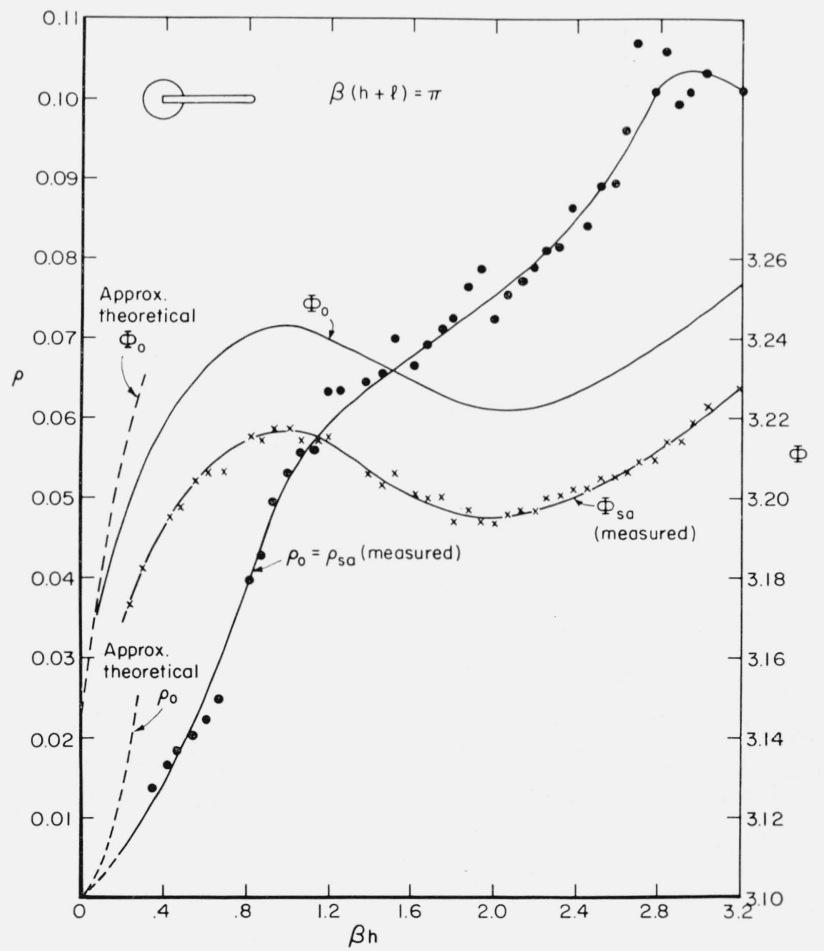

FiguRE 2. Terminal functions for inverted L-antenna, $\beta(\mathrm{h}+\mathrm{l})$ $=3.2$. 


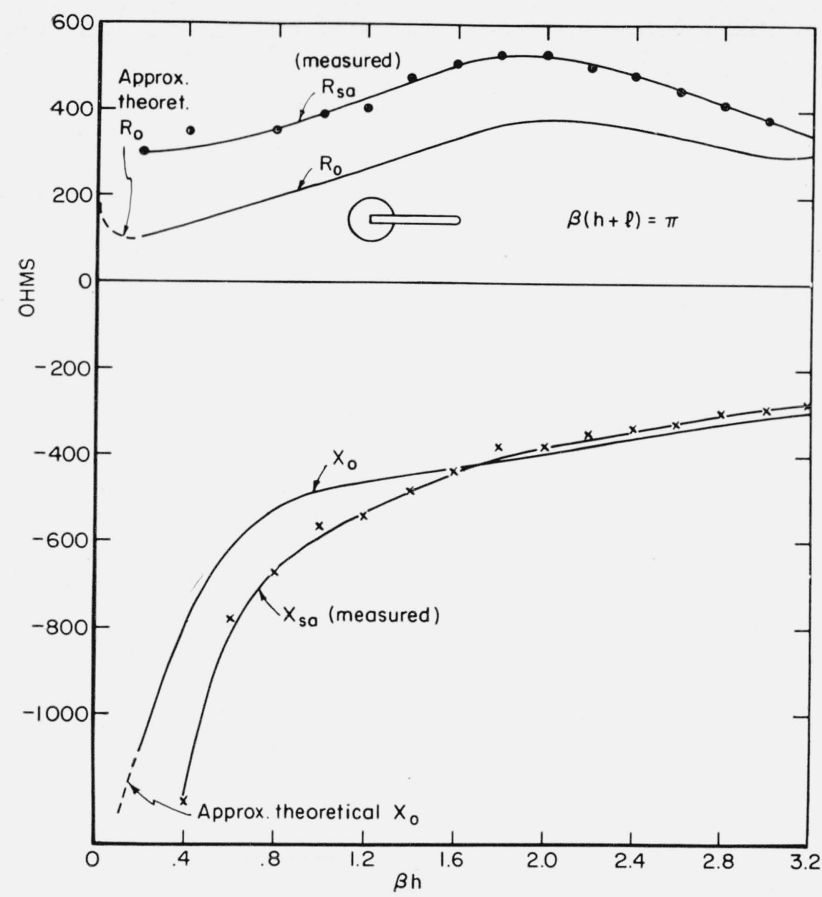

Figure 3. Impedances of inverted L-antenna, $\beta(\mathrm{h}+\mathrm{l})=3.2$.

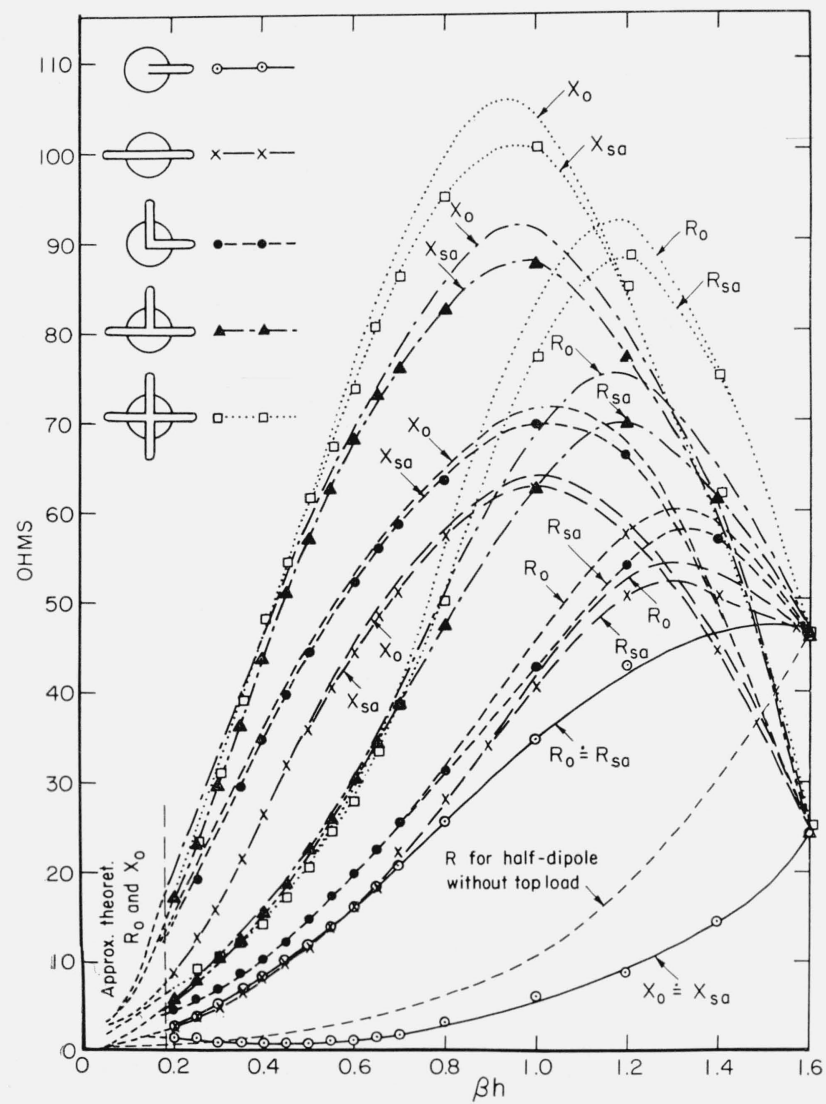

FIgure 4. Impedances of inverted L-, T-, and multi-element top-loaded antennas, $\beta(\mathrm{h}+\mathrm{l})=1.6$. binations of the two transmission-line sections in the form

$$
X_{i n}=X_{L}+X_{i n}^{\prime} ; X_{i n}^{\prime}=\frac{X_{1 i n} X_{2 i n}}{X_{1 i n}+X_{2 i n}}
$$

since the impedence is simply $Z_{i n}=R_{i n}+j X_{i n}$ where $R_{i n}$ is assumed to be given by King, Harrison, and Denton [1958]. An error due to this effect may be expected whenever $X_{2 n}$ becomes large and the current in the generator is not near a maximum. The inclusion of a series inductance $X_{L}$ in (19) is itself meaningful only when the current in the generator is near a maximum owing to the fact that there is actually no point generator but rather a distribution of driving field at the end of the coaxial line that extends over the full length $h$. For purposes of comparison $X_{i n}$ is calculated both with and without $X_{L}$ and curves of both $X_{i n}$ and $X_{i n}^{\prime}$ are shown.

The shunt driven transmission line with one end open and the other terminated in a straight conductor has three variables $s_{1}, s_{2}$ and $h$. Figure 5 shows the measured impedances when $\beta s_{2}=\pi / 2$ and $\beta h=0.161$ and 0.225 . In this case the left-hand section is terminated in a straight conductor and the corresponding terminal function is given by King [1955, pp. 123-123]. The right-hand section is adjusted in length to be near a quarter wavelength long. Let $\beta s_{2}=\pi / 2-\beta \delta^{\prime}$ where $\delta^{\prime}$ is a small length. The right end is open but it is not an ideal open end.

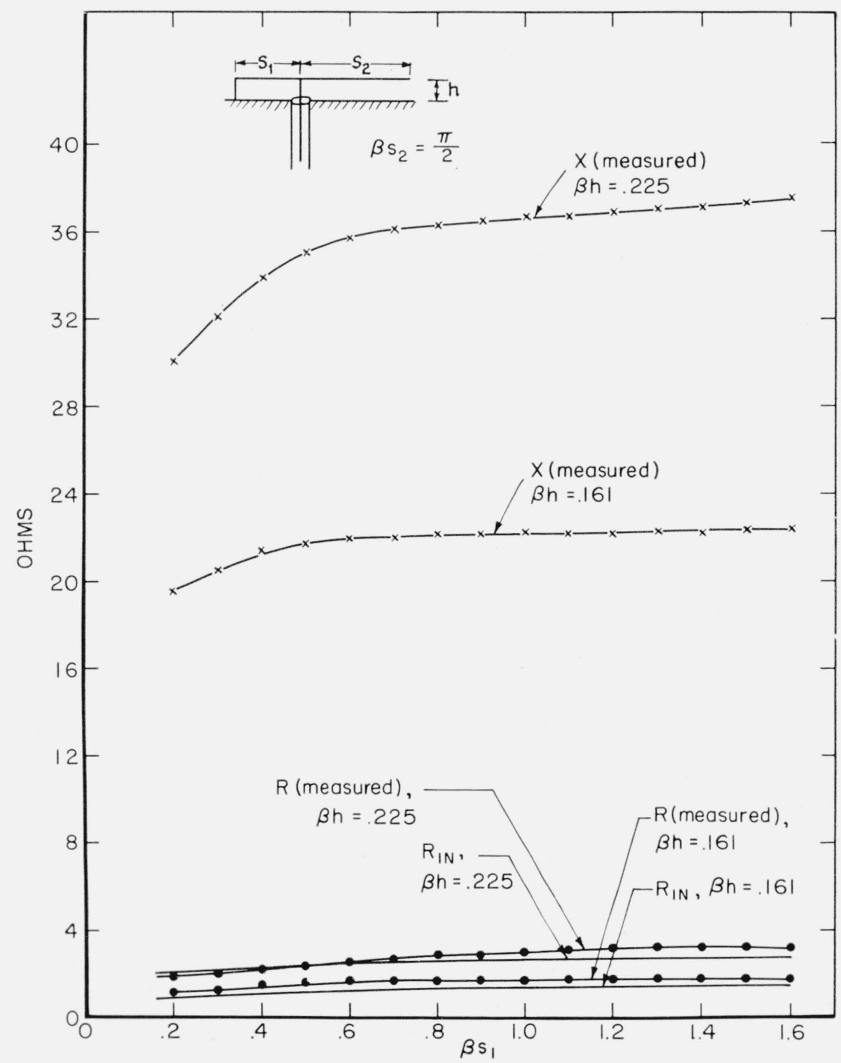

FIGURE 5. Impedances of transmission-line antennas, $\beta \mathrm{s}_{2}=\pi / 2$. 
The terminal or end effect is equivalent to a lumped capacitance $C_{T e}$ given approximately by eq (16). Tbe corresponding normalized susceptance with $R_{c}^{\prime}=120 \ln \left(2 h / a_{1}\right)$ is $b_{T e}=R_{c}^{\prime} \omega C_{T e}$. The corresponding terminal function may be obtained from King [1955, p. 119, eq 8]. The theoretical resistance curves are shown in figure 5 . It is seen that there is good agreement between the theoretical and experimental values.

The inductance of a straight section of wire of radius $a_{1}$ and length $2 h$ is given by King [1945]. The input reactances of the sections of lossless line are

$$
X_{1 i n}=-R_{c}^{\prime} \cot \left(\beta s_{1}+\Phi_{0}\right) ; X_{2 i n}=-R_{c}^{\prime} \cot \left(\beta s_{2}+\Phi_{s}\right) .
$$

For the two cases $\beta h=0.161$ and $0.225, X_{L}, X_{1 \text { in }}$ and $X_{2 i n}$ have been calculated and $X_{i n}$ determined. For the antenna over the image plane, the input reactance is $X_{i n} / 2$. The theoretical values are

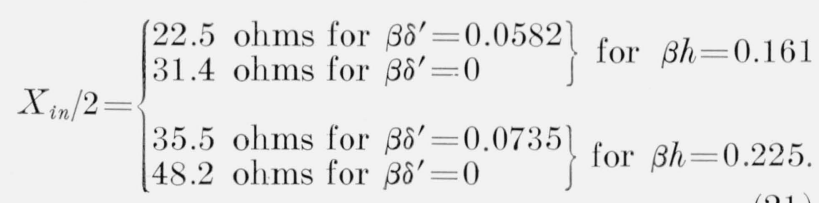

The experimentally observed values are

$$
X_{i n} / 2=\left\{\begin{array}{l}
22.3 \mathrm{ohms} \text { for } \beta h=0.161 \\
37.0 \mathrm{ohms} \text { for } \beta h=0.225 .
\end{array}\right.
$$

The value for $\beta h=0.161$ in (22) corresponds closely to the theoretical value for $\beta \delta^{\prime}=0.0582$ in $(21)$; in this case $\beta s_{2}=1.511$ or $s_{2}=0.242 \lambda$. Similarly the experimental value for $\beta h=0.225$ agrees well with the theoretical value for $s_{2}=0.245 \lambda$. The input reactance as a function of $\beta s_{1}$ is evidently very sensitive to the particular setting of $s_{2}$. A very small change in $s_{2}$ alters $X_{i n}$ significantly. The theoretical expressions involve formulas for end effects that provide corrections that are comparable with the line spacing but not comparable with the radius of the conductors. Evidently changes in $\beta s_{2}$ of the order of magnitude of $\beta a_{1}=0.025$ or small multiples thereof are precisely the order of magnitude of the difference between the observed and calculated values.

Figure 6 shows the impedance of the same structure for $\beta h=0.161$ and $\beta s_{1}=0.44$ when $s_{2}$ is varied. Resonance occurs near $\beta\left(s_{1}+s_{2}\right)=\pi / 2$. The theoretical resistance $R_{\text {in }}$ and reactance $X_{\text {in }}$ are also shown. Evidently the length $\beta\left(s_{1}+s_{2}\right)$ is a critical quantity in the manner discussed in the preceding special case with $\beta s_{2}=\pi / 2$.

The measured and theoretical impedances of the shunt-driven transmission line antenna with both ends terminated in straight conductors ( $m$-antenna) are shown in figure 7 when $\beta h=0.225$ and $\beta s_{2}=\pi / 2$. It is again evident that the setting of $\beta s_{2}$ is critical.

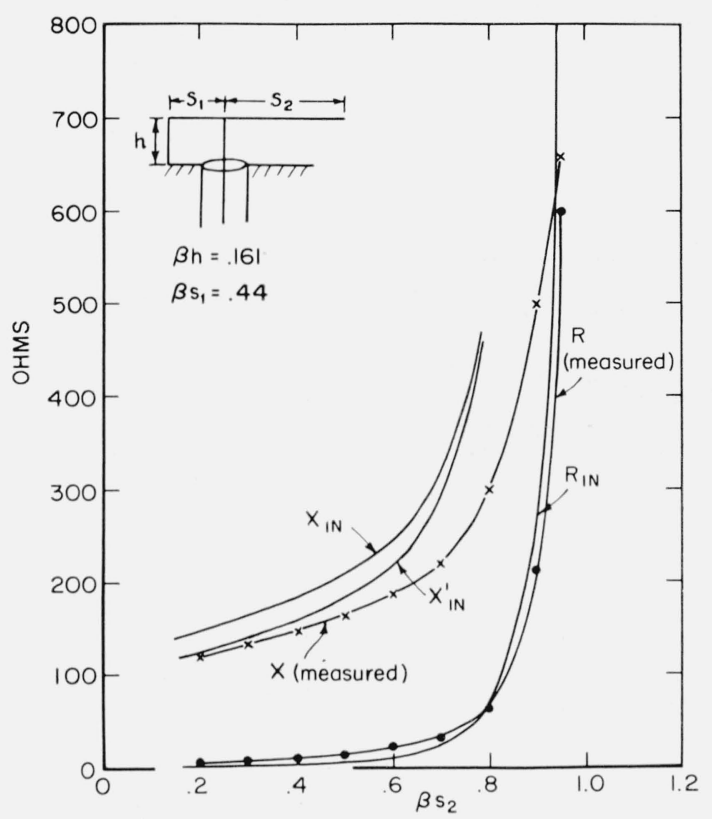

Figure 6. Impedances of transmission-line antennas, $\beta \mathrm{S}_{1}=$ $0.44, \beta \mathrm{h}=0.161$.

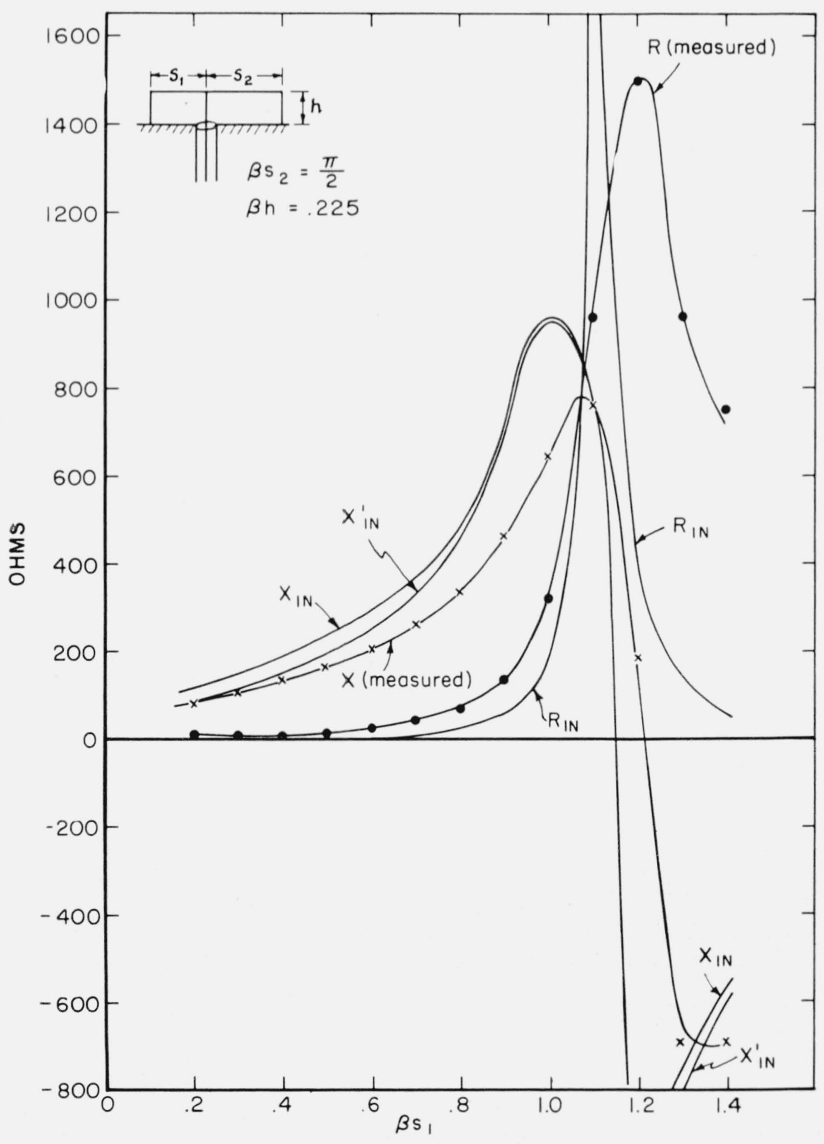

Figure 7. Impedances of $\mathrm{m}$-antenna, $\beta \mathrm{S}_{2}=\pi / 2, \beta \mathrm{h}=0.225$. 
A more detailed version of this paper appeared as Technical Report No. 305, Cruft Laboratory, Harvard University.

\section{References}

Harrison, C. W., Jr., Antenna synthesis, Sandia Corp. Tech. Memo. 37-58 (14) (March 1958a).

Harrison, C. W., Jr., Theory of inverted L-antenna with image, Sandia Corp. Tech. Memo. 11-58 (14) (April 1958b).

Harrison, C. W., Jr., Antenna analysis by circuit superposition, Sandia Corp. Tech. Memo. 250-58 (14) (June 1958c).

Harrison, C. W., Jr., Impedance of a T-antenna, Sandia Corp. Tech. Memo. 257-58 (14) (July 1958d).
King, D. D., Measurements at Centimeter Wavelength, p. 194 (D. Van Nostrand Co., Inc., New York, N.Y., 1952).

King, R. W. P., Electromagnetic Engineering, p. 433, eq. 20 (McGraw-Hill Book Co., Inc., New York, N.Y., 1945).

King, R. W. P., Transmission-line Theory (McGraw-Hill Book Co., Inc., New York, N.Y., 1955).

King, R. W. P., and C. W. Harrison, Jr., The T-antenna: current and impedance, Sandia Corp. Tech. Memo. 30659 (14) (Sept. 1959a).

King, R. W. P., and C. W. Harrison, Jr., The inverted L-antenna: current and impedance, Sandia Corp. Tech. Memo. 353-59 (14) (Nov. 1959b).

King, R. W. P., C. W. Harrison, Jr., and D. H. Denton, Jr., Transmission-line missile antennas, Sandia Corp. Tech. Memo. 436-58 (14) (Nov. 1958).

(Paper 65D5-150) 